\title{
A complex suicide
}

\section{R Ruwanpura ${ }^{1}$}

(Index words: complex suicide, electrocution, alcoholism)

\section{Introduction}

Cases with multiple simultaneous suicidal attempts are known as complex suicides. Any suicide with more than two types of injury are potentially suspicious in the manner of death [1]. Complex suicidal attempts often result in bizarre injury patterns and may simulate death due to homicide. The com-parative analysis of details of anamnesis, which must be crosschecked with another independent source and findings at the scene are vital in making conclusions about the cause of death.

\section{Case report and autopsy findings}

A 46-year old man, a chronic alcoholic and a casual labourer was pronounced dead at the emergency care unit. According to the statement given by the spouse, her husband returned home in the evening, while being under the influence of alcohol as usual, and had assaulted her over the poor quality of the dinner. Then he took a bottle of poison, mixed it with locally made alcohol and drank it. A few minutes later he was seen climbing an electric lamp post at the road side shouting that he wanted to kill himself. Then he had fallen from the lamp post (about $5 \mathrm{~m}$ in height).

${ }^{1}$ Judicial Medical Officer's Unit, Teaching Hospital, Karapitiya, Galle, Sri Lanka.

Correspondence: RR, e-mail <rohanr@sltnet.lk>. Received 27 February and revised version accepted 15 August 2009. Competing interests: none declared. 
Within 30 minutes he got up and scolded the neighbours. They ignored the victim's behaviour because neighbours have witnessed number of similar episodes in the past. Thereafter he made an unsuccessful attempt at drowning by jumping into a well where the water level was low. At that point police arrived at the scene, resaved and despatched him to the hospital. He was pronounced dead shortly after admission. An autopsy examination revealed a fresh flip laceration with an upward directed skin tag on the inner aspect of the left lower leg (Figure 1), a contusion over the right side of the hip, an oval shaped electric burn on the left thumb and index finger (Figure 2), a gaping laceration with an elongated electric mark on the frontal aspect of right wrist (Figure 3 ) and a greenish stain in the mouth with erosions in the oesophageal and gastric mucosa (Figure 4). The internal organs were free of injuries. The stomach contained a greenish oily substance. Paraquat was identified in the contents of the stomach and the urine by a spot test (Syngenta ${ }^{\mathrm{TM}}$ paraquat test kit). Early cirrhosis was noted in the liver. Lungs showed severe pulmonary oedema and parenchymal haemorrrhages. The brain was oedematous and weighed $1400 \mathrm{~g}$. Blood alcohol level was $1.85 \mathrm{~g} / 1$.

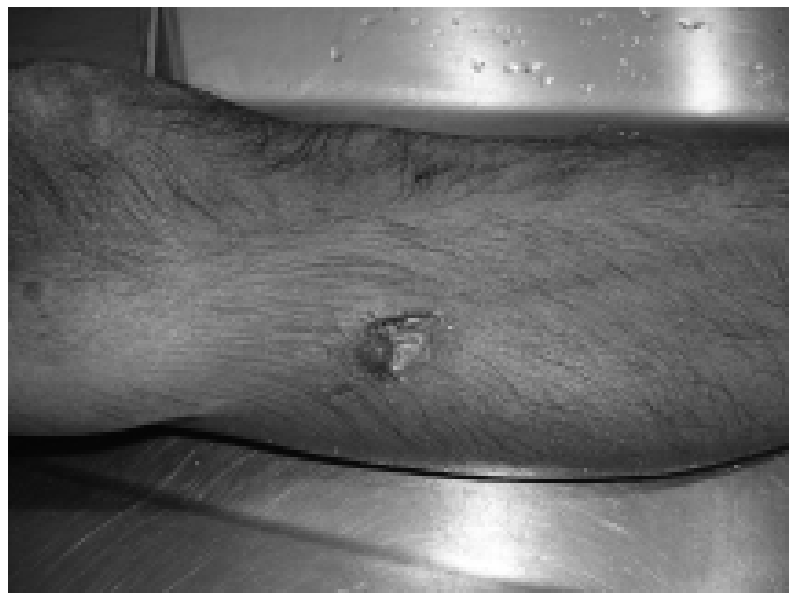

Figure 1. Laceration of the lower limb.

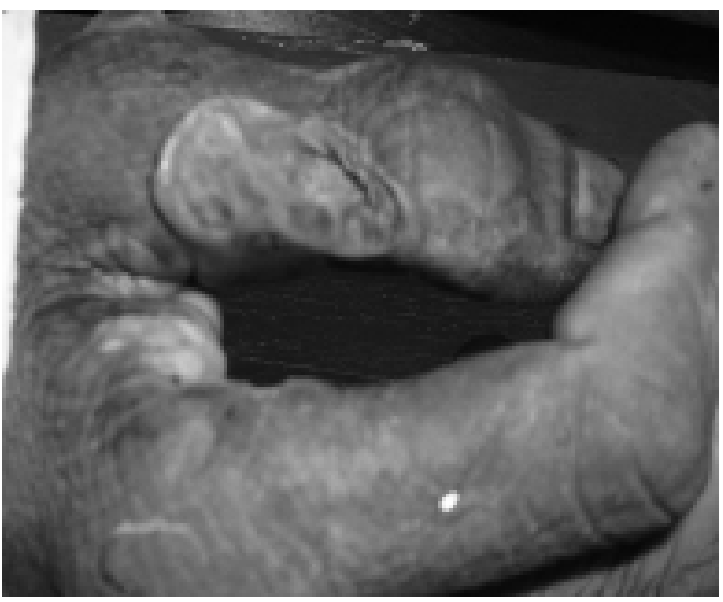

Figure 1. Electric burns of right thumb and index finger.

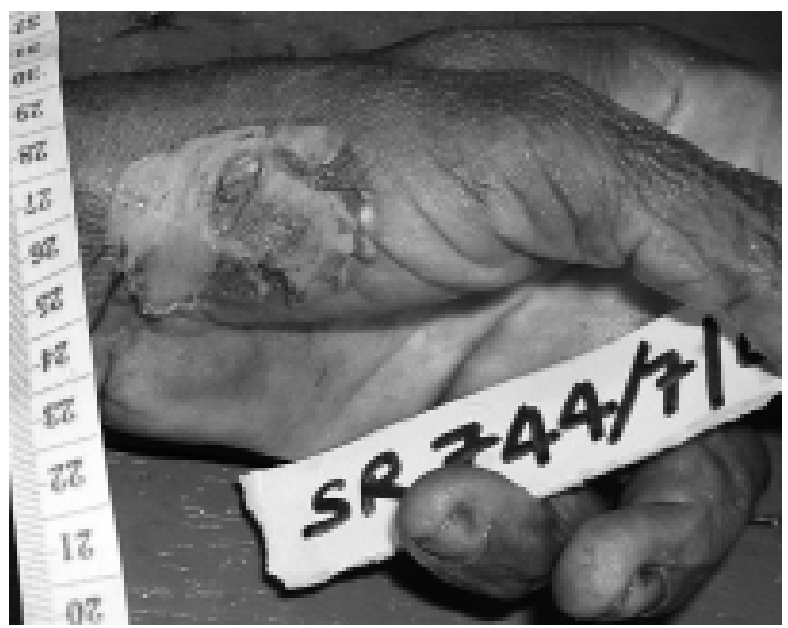

Figure 3. Electric burns on the left hand.

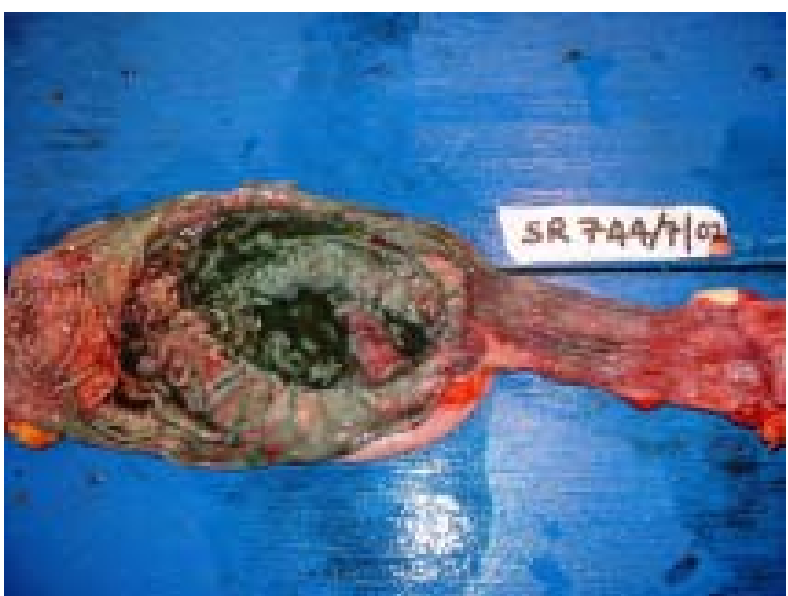

Figure 4. Oesophageal erosions and greenish staining of the gastric mucosa.

\section{Discussion}

Complex suicidal attempts often result in extraordinary injury patterns and the determination of actual cause and manner of death becomes the main task of forensic investigations [2]. The autopsy findings in this case are indicative of acute intoxication with paraquat. The positive identification of paraquat in the stomach contents and urine is adequate proof of intoxication and there is no need for blood analysis [2]. The ingestion of about $50 \mathrm{ml}$ of $21 \%$ paraquat solution (Gramoxone) is always fatal either by acute toxic effects to the lungs and brain [3] or due to development of fibrosing alveolitis within two to three weeks [4]. Hence intoxication with paraquat was given as the immediate cause of death. Though the injury pattern of this case makes the violent interference into death a remote possibility, the presence of different types of injuries on the body helped to identify the cause of death. 
Our approach to the manner of death was based on comparative study of circumstantial data with autopsy findings. Except under exceptional circumstances deaths due to ingestion of agrochemicals are generally suicidal in Sri Lanka [5]. The link between suicidal intent and alcohol dependence is well established [6]. The suicidal intent of this person could have been influenced by social problems and chronic alcoholism. Alcohol related mental disorders are a common underlying feature of suicidal actions [6] especially in bizarre cases of this nature. The victim's irrational behaviour could be explained by chronic and acute alcohol intoxication. His actions favour an unplanned complex suicide.

The injuries noted on the lower limbs could have been caused by grazing against the rough surface and in keeping with the fall from a lamp post. Injuries on the palms were typical electric burns, so called "joule burns" caused by entry or exit of the electric current. The presence of well formed electric marks on both upper limbs is sufficient to establish the cause of death as electrocution or a late arrhythmia following electrocution but the victim had completely recovered from effects of electric shock as well as impact due to the fall and had performed further volitional activities.

The multifaceted simultaneous suicidal attempts often result in complex injury patterns on the victim's body and may appear as a homicidal death. The comparative analytical approach to evaluate the origin of the injury pattern against the circumstantial evidence could answer the medico-legal queries pertaining to complex cases of this nature.

\section{References}

1. Parra MW, Lube MW, Al-Khayat H, Cheatham ML. A planned complex suicide: need for high index of suspicion. The American Surgeon 2006; 72: 454-5.

2. Lasczkowski G, Röhrich J, Bratzke H. Suicidal excess presentation of an unusual case. Archiv fur Kriminologie 1998; 202: 100-7.

3. Dasta JF. Paraquat poisoning: a review. American Journal of Hospital Pharmacy 1978; 35: 1368- 4.

4. Dinis-Olivera RJ, Duarte JA, Sanches-Navarro A, et al. Paraquat poisoning: mechanisms of lung toxicity, clinical features, and treatment. Critical Reviews in Toxicology 2008; 38: 13-57.

5. Eddleston M, Gunnell D, Karunaratne A, et al. Physical vulnerability and fatal self harm in elderly. British Journal of Psychiatry 2005; 187: 583- 4.

6. Forman EM, Berk MS, Henriques GR, Brown GK, Beck AT. History of multiple suicide attempts as a behavioural marker of severe psychopathology. American Journal of Psychiatry 2004; 161: 437-7. 